\title{
Mean Square Consistency On Numerical Solutions of Stochastic Wave Equation with Cubic Nonlinearities on 2D Rectangles
}

\author{
Haziem M. Hazaimeh \\ Department of Mathematics and statistics, Zayed University, Dubai, UAE
}

\begin{abstract}
Abstract. In this article we study the mean square consistency on numerical solutions of stochastic wave equations with cubic nonlinearities on two dimensional rectangles. In [8], we proved that the strong Fourier solution of these semi-linear wave equations exists and is unique on an appropriate Hilbert space. A linear-implicit Euler method is used to discretize the related Fourier coefficients. We prove that the linear-implicit Euler method applied to a solution of nonlinear stochastic wave equations in two dimensions is mean square consistency under the geometric condition.
\end{abstract}

\section{INTRODUCTION}

In this article we study the linear-implicit Euler method for the numerical solution of semi-linear stochastic wave equations

$$
u_{t t}=\sigma^{2} \triangle u+A\left(u, u_{t}\right)+B\left(u, u_{t}\right) \frac{d W}{d t}
$$

with cubic nonlinearities in two dimensions in terms of all systems parameters, i.e., with non-global Lipschitz continuous nonlinearities. Our study focuses on numerical solution using linear-implicit Euler method (LIEM) under the geometric condition

$\sigma^{2} \pi^{2}\left(\frac{1}{l_{x}^{2}}+\frac{1}{l_{y}^{2}}\right)-a_{1}=\frac{\sigma^{2} \pi^{2}\left(l_{x}^{2}+l_{y}^{2}\right)-a_{1} l_{x}^{2} l_{y}^{2}}{l_{x}^{2} l_{y}^{2}}:=\gamma>0$,

where $0 \leq x \leq l_{x}$ and $0 \leq y \leq l_{y}$ such that $\mathbb{D}=\left[0, l_{x}\right] \times\left[0, l_{x}\right]$. (Note that $l_{x}$ and $l_{y}$ are the dimension parameters of a vibrating plate or a membrane). We shall impose the following boundary conditions: (note that $t>0$ )

TABLE I: Boundary Conditions

\begin{tabular}{||c||c|c||c||c||c||}
\hline \hline$l_{1}$ & $u(x, 0, t)=0$ & or & $u_{x}(x, 0, t)=0$ & or & $u_{y}(x, 0, t)=0$ \\
\hline$l_{2}$ & $u\left(l_{x}, y, t\right)=0$ & or & $u_{x}\left(l_{x}, y, t\right)=0$ & or & $u_{y}\left(l_{x}, y, t\right)=0$ \\
\hline$l_{3}$ & $u\left(x, l_{y}, t\right)=0$ & or & $u_{x}\left(x, l_{y}, t\right)=0$ & or & $u_{y}\left(x, l_{y}, t\right)=0$ \\
\hline$l_{4}$ & $u(0, y, t)=0$ & or & $u_{x}(0, y, t)=0$ & or & $u_{y}(0, y, t)=0$ \\
\hline \hline
\end{tabular}

where $l_{1}, l_{2}, l_{3}$, and $l_{4}$ as in the following figure

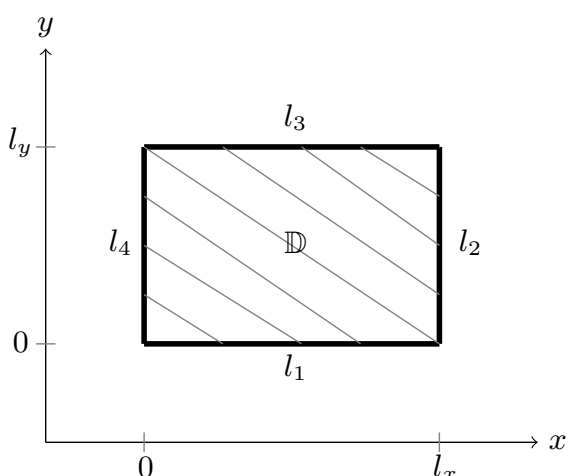

Also the initial conditions are $u(x, y, 0)=f(x, y)$ with $f \in \mathbb{L}^{2}$ (initial position) and $u_{t}(x, y, 0)=g(x, y)$ with $g \in \mathbb{L}^{2}$ (initial velocity). Recall that

$$
\mathbb{L}^{2}(\mathbb{D})=\left\{f:\left.\mathbb{D} \rightarrow \mathbb{R}\left|\int_{\mathbb{D}}\right| f(x, y)\right|^{2} d \mu(x, y)<\infty\right\},
$$

where $\mu$ is the Lebesgue measure in two dimensions.

There is a little information about the numerical solution of nonlinear stochastic wave equations. However, Schurz [17] proved that the linear-implicit method for the nonlinear stochastic heat equation just in $\mathbb{R}$ is mean square consistent with rate $r_{0}=1.5$. Also, Higham [11] studied the mean square stability of stochastic theta method and plotted the mean square stability when the test equation has real parameters. Higham, D.J., Mao, X., Stuart, A.M. [12] proved that strong convergence results but they took less restrictive conditions. To my information and since there are a few researcher work on mean square consistent of LIEM of nonlinear stochastic wave equations in two dimensions, which make me interested in working that.

Consider the stochastic nonlinear wave equation with both additive and multiplicative noise

$$
\begin{aligned}
u_{t t}= & \sigma^{2}\left(u_{x x}+u_{y y}\right)+\left(a_{1}-a_{2}\|u\|_{\mathbb{L}^{2}(\mathbb{D})}^{2}\right) u-\kappa v \\
& +\left(b_{0}+b_{1}\|u\|_{\mathbb{L}^{2}(\mathbb{D})}+b_{2}\|v\|_{\mathbb{L}^{2}(\mathbb{D})}\right) \frac{d W}{d t}
\end{aligned}
$$

with $v=u_{t}$, where

$$
W(x, y, t)=\sum_{i, j=1}^{2} \sum_{n, m=1}^{\infty} \alpha_{n, m}^{i, j} W_{n, m}^{i, j}(t) e_{n}^{i}(x) e_{m}^{j}(y)
$$

and $e_{n}^{i, j}$ and $e_{m}^{i, j}$ are orthonormalized eigenfunctions of Laplace operator $\Delta$ on $\mathbb{D}=\left[0, l_{x}\right] \times\left[0, l_{y}\right]$, driven by i.i.d. standard Wiener processes $W_{n, m}^{i, j}$ with $\mathbb{E}\left[W_{n, m}^{i, j}(t)\right]=0, \mathbb{E}\left[W_{n, m}^{i, j}(t)\right]^{2}=t$. Recall 
that $e_{n}^{i}(z)$ and $e_{m}^{j}(z)$ are one of the functions $\sqrt{\frac{2}{l_{z}}} \sin \left(k \pi z / l_{z}\right)$ or $\sqrt{\frac{2}{l_{z}}} \cos \left(k \pi z / l_{z}\right)$, with $z=x$ or $y, k=n$ or $m$, and $i, j=1,2$. The solution of equation (1) in term of Fourier series is

$$
u(x, y, t)=\sum_{i, j=1}^{2} \sum_{n, m=1}^{\infty} c_{n, m}^{i, j}(t) e_{n}^{i}(x) e_{m}^{j}(y) .
$$

The form of Fourier solutions $u$ and its approximate Fourier solutions $u_{N}$ given by

$$
u_{N}(x, y, t)=\sum_{i, j=1}^{2} \sum_{n, m=1}^{N} c_{n, m}^{i, j}(t) e_{n}^{i}(x) e_{m}^{j}(y)
$$

with its coefficients $c_{n, m}^{i, j}$ satisfying

$$
\begin{aligned}
\ddot{c}_{n, m}^{i, j}(t)= & {\left[-\sigma^{2}\left(\lambda_{n}+\beta_{m}\right)+a_{1}-a_{2}\|u\|_{\mathbb{L}^{2}(\mathbb{D})}^{2}\right] c_{n, m}^{i, j}(t) } \\
& -\kappa v_{n, m}^{i, j}(t)+\left(b_{0}+b_{1}\|u\|_{\mathbb{L}^{2}(\mathbb{D})}\right. \\
& \left.+b_{2}\|\dot{u}\|_{\mathbb{L}^{2}(\mathbb{D})}\right) \alpha_{n, m}^{i, j} \dot{W}_{n, m}^{i, j}(t)
\end{aligned}
$$

where $\lambda_{n}=\frac{\pi^{2} n^{2}}{l_{x}^{2}}, \quad \beta_{m}=\frac{\pi^{2} m^{2}}{l_{y}^{2}},\|u\|_{\mathbb{L}^{2}(\mathbb{D})}=$ $\sqrt{\sum_{i, j=1}^{2} \sum_{n, m=1}^{N}\left[c_{n, m}^{i, j}\right]^{2}}$, and $\|\dot{u}\|_{\mathbb{L}^{2}(\mathbb{D})}=\sqrt{\sum_{i, j=1}^{2} \sum_{n, m=1}^{N}\left[\dot{c}_{n, m}^{i, j}\right]^{2}}$. Note that equation (4) is equivalent to the following system

$$
\begin{aligned}
\dot{c}_{n, m}^{i, j}= & v_{n, m}^{i, j} \\
\dot{v}_{n, m}^{i, j}= & {\left[-\sigma^{2}\left(\lambda_{n}+\beta_{m}\right)+a_{1}-a_{2} \sum_{i, j}^{2} \sum_{k, l=1}^{N}\left[c_{k, l}^{i, j}\right]^{2}\right] c_{n, m}^{i, j} } \\
& -\kappa v_{n, m}^{i, j}(t) \\
& +\left(b_{0}+b_{1}\|u\|_{\mathbb{L}^{2}(\mathbb{D})}+\|\dot{u}\|_{\mathbb{L}^{2}(\mathbb{D})}\right) \alpha_{n, m}^{i, j} \dot{W}_{n, m}^{i, j} .
\end{aligned}
$$

subject to the initial conditions

$$
\left(c_{n, m}^{i, j}(0), v_{n, m}^{i, j}(0)\right)=\left(c_{n, m, 0}^{i, j}, v_{n, m, 0}^{i, j}\right)_{n, m \geq 1, i, j=1,2} .
$$

The solution of system (5) is exist and unique [8]. Now, we introduce the following standard definitions.

Definition 1: For $k \in \mathbb{N}$, take the partition $0=t_{0}<t_{1}<t_{2}<$ $\ldots<t_{k}=T$ of $[0, T]$ with current step sizes $h_{k}=t_{k+1}-t_{k}>$ 0 , then , as in Schurz [18], the linear-implicit Euler-type method (LIEM) is governed by the iterative scheme

$$
\begin{aligned}
c_{n, m}^{i, j}\left(t_{k+1}\right)= & c_{n, m}^{i, j}\left(t_{k}\right)+h_{k} v_{n, m}^{i, j}\left(t_{k+1}\right) \\
v_{n, m}^{i, j}\left(t_{k+1}\right)= & v_{n, m}^{i, j}\left(t_{k}\right)+h_{k}\left[f_{n, m}\left(u\left(t_{k}\right)\right) c_{n, m}^{i, j}\left(t_{k+1}\right)\right. \\
& \left.-\kappa v_{n, m}^{i, j}\left(t_{k+1}\right)\right]+g_{n, m}\left(u\left(t_{k}\right)\right) \triangle_{k} W_{n, m}^{i, j}
\end{aligned}
$$

where

$$
f_{n, m}\left(u\left(t_{k}\right)\right)=-\sigma^{2}\left(\lambda_{n}+\beta_{m}\right)+a_{1}-a_{2} \sum_{i, j=1}^{2} \sum_{n, m=1}^{N}\left[c_{n, m}^{i, j}\left(t_{k}\right)\right]^{2}
$$

and

$$
\begin{aligned}
g_{n, m}\left(u\left(t_{k}\right)\right) & =\left(b_{0}+b_{1} \sqrt{\sum_{i, j=1}^{2} \sum_{n, m=1}^{N}\left(c_{n, m}^{i, j}\left(t_{k}\right)\right)^{2}}\right. \\
& +b_{2} \sqrt{\left.\sum_{i, j=1}^{2} \sum_{n, m=1}^{N}\left(\dot{c}_{n, m}^{i, j}\left(t_{k}\right)\right)^{2}\right)} \alpha_{n, m}^{i, j}
\end{aligned}
$$

and

$\triangle_{k} W_{n, m}^{i, j}=W_{n, m}^{i, j}\left(t_{k+1}\right)-W_{n, m}^{i, j}\left(t_{k}\right) \in \mathcal{N}\left(0, h_{k}\right), h_{k}=t_{k+1}-t_{k}$.
In [10], we proved that the explicit representation of LIEM as in the following theorem

Theorem 1: Assume that $\left|c_{n, m}^{i, j}\left(t_{k}\right)\right|<\infty,\left|v_{n, m}^{i, j}\left(t_{k}\right)\right|<\infty$, and $a_{2} \geq 0, \kappa \geq 0$, and

$$
\forall n, m \in \mathbb{N}:\left[-\sigma^{2}\left(\lambda_{n}+\beta_{m}\right)+a_{1}\right] h_{k}^{2}<1+h_{k} \kappa .
$$

Then the method (LIEM) governed by equation (6) and equation (7) has the non-exploding explicit representation

$$
\text { 1) } \begin{aligned}
c_{n, m}^{i, j}\left(t_{k+1}\right)=\quad & \frac{\left(1+h_{k} \kappa\right) c_{n, m}^{i, j}\left(t_{k}\right)+h_{k} v_{n, m}^{i, j}\left(t_{k}\right)}{1+h_{k} \kappa-h_{k}^{2} f_{n, m}\left(u\left(t_{k}\right)\right)} \\
& +\frac{h_{k} g_{n, m}\left(u\left(t_{k}\right) \triangle_{k} W_{n, m}^{i, j}\right.}{1+h_{k} \kappa-h_{k}^{2} f_{n, m}\left(u\left(t_{k}\right)\right)}
\end{aligned}
$$

and

$$
\begin{array}{r}
2) v_{n, m}^{i, j}\left(t_{k+1}\right)=\frac{v_{n, m}^{i, j}\left(t_{k}\right)+h_{k} f_{n, m}\left(u\left(t_{k}\right)\right) c_{n, m}^{i, j}\left(t_{k}\right)}{1+h_{k} \kappa-h_{k}^{2} f_{n, m}\left(u\left(t_{k}\right)\right)} \\
+\frac{g_{n, m}\left(u\left(t_{k}\right)\right) \triangle_{k} W_{n, m}^{i, j}}{1+h_{k} \kappa-h_{k}^{2} f_{n, m}\left(u\left(t_{k}\right)\right)}
\end{array}
$$

where $f_{n, m}\left(u\left(t_{k}\right)\right), g_{n, m}\left(u\left(t_{k}\right)\right), \triangle_{k} W_{n, m}^{i, j}$, and $h_{k}$ as above.

Proof 2: (Theorem 1) See [10]

\section{Mean Square Consistency of Numerical Methods}

Definition 2: A numerical method with the scheme

$$
\hat{u}(t+h)=\hat{u}(t)+h f(\hat{u}(t))+g(\hat{u}(t)) \triangle W(t)
$$

where $h=t_{k+1}-t_{k}=\int_{t_{k}}^{t_{k+1}} d s, \Delta W^{k}=W\left(t_{k+1}\right)-$ $W\left(t_{k}\right)=\int_{t_{k}}^{t_{k+1}} d W(s) \in \mathcal{N}(0, h)$ and $f(u(t))$ and $g(u(t))$ as above applied to SDE (5) is said to be mean square consistent with rate $r_{2}>0$ on $\mathbb{D}$ iff $\exists K_{2}^{c}=$ consistency constant $\forall 0 \leq t \leq$ $t+h \leq T$ where $\mathrm{h}$ is sufficiently small, i.e., $0<h \leq \delta \leq 1$, $\forall u(t) \in H$, where $H:=\left\{u \in \mathbb{L}^{2}(\mathbb{D}) \mid \dot{u} \in \mathbb{L}^{2}(\mathbb{D})\right\}$, and $\|u\|_{H}=\sqrt{\|u\|_{\mathbb{L}^{2}(\mathbb{D})}^{2}+\|\dot{u}\|_{\mathbb{L}^{2}(\mathbb{D})}^{2}}:\left(\mathcal{F}_{t}, \mathcal{B}\left(\mathbb{L}^{2}\right)\right)-$ measurable, then

$$
\left(\mathbb{E}\left[\|u(t+h)-\hat{u}(t+h)\|_{N \times N}^{2} \mid \mathcal{F}_{t}\right]\right)^{\frac{1}{2}} \leq K_{2}^{c}(V(u(t))) h^{r_{2}}
$$

where

$$
\hat{u}(t+h \mid t, u(t))=\hat{u}(t)+\int_{t}^{t+h} f(u(s)) d s+\int_{t}^{t+h} g(u(s)) d W(s) d s
$$

Lemma 3: (The Burkholder-Davis-Gundy Inequality)

$$
\mathbb{E}\left\|\int_{0}^{t} g(s) d W(s)\right\|^{p} \leq C_{B} \mathbb{E}\left(\int_{0}^{t}\|g(s)\|^{2} d s\right)^{\frac{p}{2}}
$$

Proof 4: (Lemma 1) See Karatzas and Shreve [13].

Lemma 5: Let $X \sim \mathcal{N}\left(0, h_{k}\right), \forall n \in \mathbb{N}, n \geq 1$, we have

$$
\mathbb{E}(X)^{2 n}=(2 n-1) ! ! h_{k}^{n},
$$

where $(2 n-1) ! !=(2 n-1) \times(2 n-3) \times(2 n-5) \times \ldots \times 5 \times 3 \times 1$.

Proof 6: (Lemma 2) See [9].

Theorem 7: The method (LIEM) given by

$$
\begin{gathered}
c_{n, m}^{i, j}\left(t_{k+1}\right)=c_{n, m}^{i, j}\left(t_{k}\right)+h_{k} v_{n, m}^{i, j}\left(t_{k+1}\right) \\
v_{n, m}^{i, j}\left(t_{k+1}\right)=v_{n, m}^{i, j}\left(t_{k}\right)+h_{k}\left[f_{n, m}\left(u\left(t_{k}\right)\right) c_{n, m}^{i, j}\left(t_{k+1}\right)\right. \\
\left.-\kappa v_{n, m}^{i, j}\left(t_{k+1}\right)\right]+g_{n, m}\left(u\left(t_{k}\right)\right) \triangle_{k} W_{n, m}^{i, j}
\end{gathered}
$$

is locally mean square consistent with rate $r_{2} \geq 1$, where $f\left(u\left(t_{k}\right)\right)$ and $g\left(u\left(t_{k}\right)\right)$ as above and

$\triangle_{k} W_{n, m}^{i, j}=W_{n, m}^{i, j}\left(t_{k+1}\right)-W_{n, m}^{i, j}\left(t_{k}\right) \in \mathcal{N}\left(0, h_{k}\right), h_{k}=t_{k+1}-t_{k}$ 
Proof 8: (Theorem 2) We want to prove that

1) $\left(\mathbb{E}\left[\|c(t+h)-\hat{c}(t+h)\|_{N}^{2} \mid \hat{u}(t)\right]\right)^{\frac{1}{2}} \leq K_{c_{2}}^{c} V(u(t)) h^{r_{2}}$

$$
2) \mathbb{E}\left[\left\|v_{n, m}(t+h)-\hat{v}_{n, m}(t+h)\right\|_{N}^{2} \hat{u}(t)\right)^{\frac{1}{2}} \leq K_{v_{2}}^{c} V(u(t)) h^{r_{2}}
$$

Provided that $c(t)=\hat{c}(t)$ and $v(t)=\hat{v}(t)$. To prove inequality (14), we know from [10] that

$$
\begin{aligned}
c(t+h)-\hat{c}(t+h)= & \int_{t}^{t+h}\left(v(s)-\hat{f}_{c}(u(t))\right) d s \\
& -\int_{t}^{t+h} \hat{g}_{c}(u(t)) d W(s) .
\end{aligned}
$$

Thus,

$$
\begin{aligned}
\left\|c_{n, m}(t+h)-\hat{c}_{n, m}(t+h)\right\|_{N}^{2} \\
=\left\|\int_{t}^{t+h}\left(v(s)-\hat{f}_{c}(u(t))\right) d s-\int_{t}^{t+h} \hat{g}_{c}(u(t)) d W(s)\right\|_{N}^{2} \\
\leq 2\left\|\int_{t}^{t+h}\left(v(s)-\hat{f}_{c}(u(t))\right) d s\right\|_{N}^{2} \\
\quad+2\left\|\int_{t}^{t+h} \hat{g}_{c}(u(t)) d W(s)\right\|_{N}^{2}
\end{aligned}
$$

by using the fact that $(a+b)^{2} \leq 2 a^{2}+2 b^{2}$. So

$$
\begin{aligned}
\mathbb{E}\|c(t+h)-\hat{c}(t+h)\|_{N}^{2} & \leq 2 \mathbb{E}\left\|\int_{t}^{t+h}\left(v(s)-\hat{f}_{c}(u(t))\right) d s\right\|_{N}^{2} \\
& +2 \mathbb{E}\left\|\int_{t}^{t+h} \hat{g}_{c}(u(t)) d W(s)\right\|_{N}^{2}
\end{aligned}
$$

which implies that

$$
\begin{aligned}
\mathbb{E}\|c(t+h)-\hat{c}(t+h)\|_{N}^{2} & \leq 2 \int_{t}^{t+h} \mathbb{E}\left\|v(s)-\hat{f}_{c}(u(t))\right\|_{N}^{2} d s \\
& +2 \mathbb{E}\left\|\int_{t}^{t+h} \hat{g}_{c}(u(t)) d W(s)\right\|_{N}^{2}
\end{aligned}
$$

First, we will find the first part of the right hand side of inequality (16),

$$
\begin{gathered}
\left\|v(s)-\hat{f}_{c}(u(t))\right\|_{N}^{2}=\left\|v(s)-v(t)+v(t)-\hat{f}_{c}(u(t))\right\|_{N}^{2} \\
\leq 2\|v(s)-v(t)\|_{N}^{2}+2\left\|v(t)-\hat{f}_{c}(u(t))\right\|_{N}^{2}
\end{gathered}
$$

Step 1: We know that

$$
\begin{aligned}
d v_{n, m}(r) & \\
& =\left[-\sigma^{2}\left(\lambda_{n}+\beta_{m}\right)-a_{1}+a_{2}\|u(r)\|_{\mathbb{L}^{2}(\mathbb{D})}^{2}\right] c_{n, m}(r) d r \\
& -\kappa v_{n, m}(r) d r+g(u(r)) d W_{n, m}(r)
\end{aligned}
$$

thus

$$
\begin{aligned}
v_{n, m}(s) & -v_{n, m}(t) \\
= & \int_{t}^{s}\left[-\sigma^{2}\left(\lambda_{n}+\beta_{m}\right)-a_{1}+a_{2}\|u(r)\|_{\mathbb{L}^{2}(\mathbb{D})}^{2}\right] c_{n, m}(r) d r \\
& -\kappa \int_{t}^{s} v_{n, m}(r) d r+\int_{t}^{s} g(u(r)) d W_{n, m}(r)
\end{aligned}
$$

so

$$
\begin{aligned}
\| v_{n, m}(s) & -v_{n, m}(t) \|_{N}^{2} \\
& \leq 4\left[\sigma^{2}\left(\lambda_{n}+\beta_{m}\right)-a_{1}\right]^{2}\left\|\int_{t}^{s} c_{n, m}(r) d r\right\|_{N}^{2} \\
& +4 a_{2}^{2}\left\|\int_{t}^{s}\right\| u(r)\left\|_{\mathbb{L}^{2}(\mathbb{D})}^{2} c_{n, m}(r) d r\right\|_{N}^{2} \\
& +4 \kappa^{2}\left\|\int_{t}^{s} v_{n, m}(r) d r\right\|_{N}^{2} \\
& +4\left\|\int_{t}^{s} g(u(r)) d W_{n, m}(r)\right\|_{N}^{2}
\end{aligned}
$$

therefore,

$$
\begin{aligned}
\mathbb{E} \| v_{n, m}(s) & -v_{n, m}(t) \|_{N}^{2} \\
& \leq 4\left[\sigma^{2}\left(\lambda_{n}+\beta_{m}\right)-a_{1}\right]^{2} \mathbb{E}\left\|\int_{t}^{s} c_{n, m}(r) d r\right\|_{N}^{2} \\
& +4 a_{2}^{2} \mathbb{E}\left\|\int_{t}^{s}\right\| u(r)\left\|_{\mathbb{L}^{2}(\mathbb{D})}^{2} c_{n, m}(r) d r\right\|_{N}^{2} \\
& +4 \kappa^{2} \mathbb{E}\left\|\int_{t}^{s} v_{n, m}(r) d r\right\|_{N}^{2} \\
& +4 \mathbb{E}\left\|\int_{t}^{s} g(u(r)) d W_{n, m}(r)\right\|_{N}^{2} \\
& \leq 4\left[\sigma^{2}\left(\lambda_{n}+\beta_{m}\right)-a_{1}\right]^{2} \int_{s}^{t} \mathbb{E}\|c(r)\|_{N}^{2} d r \\
& +4 a_{2}^{2} \int_{s}^{t}\left(\mathbb{E}\|u(r)\|_{\mathbb{L}^{2}(\mathbb{D})}^{8}\right)^{\frac{1}{2}}\left(\mathbb{E}\|c(r)\|_{N}^{2}\right)^{\frac{1}{2}} d r \\
& +4 \kappa^{2} \int_{s}^{t} \mathbb{E}\|v(r)\|_{N}^{2} d r \\
& +4 C_{B} \mathbb{E} \int_{s}^{t}\|g(u(r))\|_{N}^{2} d W(r)
\end{aligned}
$$

by using Hölder and Burkerholder-Davis-Gundy inequalities, Lemma 3 , then substituting

$$
g(u(t))=\left(b_{0}+b_{1}\|u(t)\|_{l^{2}}+\|v(t)\|_{l^{2}}\right) \alpha^{2},
$$

we get

$$
\begin{aligned}
& \mathbb{E}\left\|v_{n, m}(s)-v_{n, m}(t)\right\|_{N}^{2} \\
& \quad \leq 4\left(\left[\sigma^{2}\left(\lambda_{n}+\beta_{m}\right)-a_{1}\right]^{2}+\kappa^{2}\right) \mathbb{E} V(u(s))|s-t| \\
& \quad+12 C_{B} \alpha^{2}\left[b_{0}^{2}+\left(b_{1}^{2}+b_{2}^{2}\right) \mathbb{E} V(u(t))\right]|s-t| \\
& \quad+4 a_{2}^{2} \mathbb{E} V^{2}(u(t)) \mathbb{E} V(u(s))|s-t| \\
& \quad \leq \max _{1 \leq n, m \leq N} 4\left(\left[\sigma^{2}\left(\lambda_{n}+\beta_{m}\right)-a_{1}\right]^{2}+\kappa^{2}\right) \mathbb{E} V(u(s))|s-t| \\
& \quad+12 C_{B} \alpha^{2}\left[b_{0}^{2}+\left(b_{1}^{2}+b_{2}^{2}\right) \mathbb{E} V(u(t))\right]|s-t| \\
& \quad+8 a_{2} \mathbb{E} V^{2}(u(t)) \mathbb{E} V(u(t))|s-t|
\end{aligned}
$$

and, if $N$ is sufficiently large, we find

$$
\begin{aligned}
& \mathbb{E}\left\|v_{n, m}(s)-v_{n, m}(t)\right\|_{N}^{2} \\
& \quad \leq 4\left(\left[\sigma^{2} \pi^{2} N^{2}\left(\frac{1}{l_{x}^{2}}+\frac{1}{l_{y}^{2}}\right)\right]^{2}+\kappa^{2}\right) \mathbb{E} V(u(s))|s-t| \\
& \quad+12 C_{B} \alpha^{2}\left[b_{0}^{2}+\left(b_{1}^{2}+b_{2}^{2}\right) \mathbb{E} V(u(t))\right]|s-t| \\
& \quad+8 a_{2} \mathbb{E} V^{2}(u(t)) \mathbb{E} V(u(t))|s-t|
\end{aligned}
$$


taking the conditional expectation gives us

$$
\begin{aligned}
& \mathbb{E}\left(\left\|v_{n, m}(s)-v_{n, m}(t)\right\|_{N}^{2} \mid u(t)\right) \\
& \quad \leq 4\left(\sigma^{4} \pi^{4} N^{4}\left(\frac{1}{l_{x}^{2}}+\frac{1}{l_{y}^{2}}\right)^{2}+\kappa^{2}+3 C_{B} \alpha^{2}\left(b_{1}^{2}+b_{2}^{2}\right)\right) V(u(t))|s-t| \\
& \quad+12 C_{B} \alpha^{2} b_{0}^{2} V(u(t))|s-t|+8 a_{2} V^{2}(u(t)) V(u(t))|s-t| \\
& \quad \leq K_{5}(1+V(u(t)))|s-t| \\
& \quad \leq K_{5} \tilde{V}(u(t))|s-t|
\end{aligned}
$$

where

$$
\begin{aligned}
K_{5}= & 4\left(\sigma^{4} \pi^{4} N^{4}\left(\frac{1}{l_{x}^{2}}+\frac{1}{l_{y}^{2}}\right)^{2}+\kappa^{2}+3 C_{B} \alpha^{2}\left(b_{1}^{2}+b_{2}^{2}\right)\right) \\
& +12 C_{B} \alpha^{2} b_{0}^{2}+8 a_{2} V^{2}(u(t))
\end{aligned}
$$

therefore,

$$
\begin{aligned}
\int_{t}^{t+h} \mathbb{E}\left[\| v_{n, m}(s)\right. & \left.-v_{n, m}(t) \|_{N}^{2} \mid u(t)\right] d s \\
& \leq k_{5} \tilde{V}(u(t)) \frac{h_{k}^{2}}{2} \\
& \leq k_{5} \tilde{V}(u(t)) h_{k}^{2} .
\end{aligned}
$$

Step 2: Also, we know that,

$$
\begin{array}{r}
v_{n, m}(t)-\hat{f}_{c}(u(t)) \\
=\frac{\left(h_{k} \kappa+h_{k}\left[\sigma^{2}\left(\lambda_{n}+\beta_{m}\right)-a_{1}\right]\right) v_{n, m}(t)}{1+h_{k} \kappa+h_{k}^{2}\left[\sigma^{2}\left(\lambda_{n}+\beta_{m}\right)-a_{1}+a_{2} \sum_{i, j=1}^{2} \sum_{n, m=1}^{N}\left[c_{n, m}^{i, j}\left(t_{k}\right)\right]^{2}\right]} \\
+\frac{h_{k}^{2} a_{2}\left(\sum_{i, j=1}^{2} \sum_{n, m=1}^{N}\left[c_{n, m}^{i, j}\left(t_{k}\right)\right]^{2}\right) v_{n, m}(t)}{1+h_{k} \kappa+h_{k}^{2}\left[\sigma^{2}\left(\lambda_{n}+\beta_{m}\right)-a_{1}+a_{2} \sum_{i, j=1}^{2} \sum_{n, m=1}^{N}\left[c_{n, m}^{i, j}\left(t_{k}\right)\right]^{2}\right]} \\
+\frac{h_{k}\left[\sigma^{2}\left(\lambda_{n}+\beta_{m}\right)-a_{1}\right] c_{n, m}(t)}{1+h_{k} \kappa+h_{k}^{2}\left[\sigma^{2}\left(\lambda_{n}+\beta_{m}\right)-a_{1}+a_{2} \sum_{i, j=1}^{2} \sum_{n, m=1}^{N}\left[c_{n, m}^{i, j}\left(t_{k}\right)\right]^{2}\right]} \\
+\frac{h_{k} a_{2}\left(\sum_{i, j=1}^{2} \sum_{n, m=1}^{N}\left[c_{n, m}^{i, j}\left(t_{k}\right)\right]^{2}\right) c_{n, m}(t)}{1+h_{k} \kappa+h_{k}^{2}\left[\sigma^{2}\left(\lambda_{n}+\beta_{m}\right)-a_{1}+a_{2} \sum_{i, j=1}^{2} \sum_{n, m=1}^{N}\left[c_{n, m}^{i, j}\left(t_{k}\right)\right]^{2}\right]}
\end{array}
$$

to simplify, let

$$
\begin{array}{r}
F(u(t))=\quad 1+h_{k} \kappa \\
+h_{k}^{2}\left[\sigma^{2}\left(\lambda_{n}+\beta_{m}\right)-a_{1}\right] \\
+a_{2} \sum_{i, j=1}^{2} \sum_{n, m=1}^{N}\left[c_{n, m}^{i, j}\left(t_{k}\right)\right]^{2},
\end{array}
$$

which gives $F(u(t)) \geq 0$. Now, if we pulling the expectation over the squared norm of the latter identity, we find that

$$
\begin{aligned}
& \mathbb{E}\left\|v_{n, m}(t)-\hat{f}_{c}(u(t))\right\|_{N}^{2} \\
& \leq 4 h_{k}^{2}\left(\kappa+h_{k}\left[\sigma^{2}\left(\lambda_{n}+\beta_{m}\right)-a_{1}\right]\right)^{2} \mathbb{E}\left\|\frac{v(t)}{F(u(t))}\right\|_{N}^{2} \\
& +4 h_{k}^{4} a_{2}^{2} \mathbb{E}\left\|\frac{\|u(t)\|_{N}^{2} v(t)}{F(u(t))}\right\|_{N}^{2} \\
& +4 h_{k}^{2}\left[\sigma^{2}\left(\lambda_{n}+\beta_{m}\right)-a_{1}\right]^{2} \mathbb{E}\left\|\frac{c(t)}{F(u(t))}\right\|_{N}^{2} \\
& +4 h_{k}^{2} a_{2}^{2} \mathbb{E}\left\|\frac{\|u(t)\|_{N}^{2} c(t)}{F(u(t))}\right\|_{N}^{2}
\end{aligned}
$$

but we know that $F_{c}(u(t)) \geq 0$, so

$$
\begin{aligned}
\mathbb{E} \| v(t) & -\hat{f}_{c}(u(t)) \|_{N}^{2} \\
\leq \quad & 4 h_{k}^{2}\left(\kappa+h_{k}\left[\sigma^{2}\left(\lambda_{n}+\beta_{m}\right)-a_{1}\right]\right)^{2} \mathbb{E}\|v(t)\|_{N}^{2} \\
& +4 h_{k}^{4} a_{2}^{2} \mathbb{E}\|\| u(t)\left\|_{N}^{2} v(t)\right\|_{N}^{2} \\
& +4 h_{k}^{2}\left(\sigma^{2}\left(\lambda_{n}+\beta_{m}\right)-a_{1}\right)^{2} \mathbb{E}\|c(t)\|_{N}^{2} \\
& +4 a_{2}^{2} h_{k}^{2} \mathbb{E}\|\| u(t)\left\|_{N}^{2} c(t)\right\|_{N}^{2} \\
\leq \quad & 4 h_{k}^{2}\left(\kappa+h_{k}\left[\sigma^{2}\left(\lambda_{n}+\beta_{m}\right)-a_{1}\right]\right)^{2} \mathbb{E} V(u(t)) \\
& +4 h_{k}^{4} a_{2}^{2}\left(\mathbb{E}\|u(t)\|_{N}^{4}\right)^{\frac{1}{2}}\left(\mathbb{E}\|v(t)\|_{N}^{2}\right)^{\frac{1}{2}} \\
& +4 h_{k}^{2}\left[\sigma^{2}\left(\lambda_{n}+\beta_{m}\right)-a_{1}\right]^{2} \mathbb{E} V(u(t)) \\
& +4 h_{k}^{2} a_{2}^{2}\left(\mathbb{E}\|u(t)\|_{N}^{4}\right)^{\frac{1}{2}}\left(\mathbb{E}\|c(t)\|_{N}^{2}\right)^{\frac{1}{2}} \\
\leq \quad & 4 h_{k}^{2}\left(\kappa+h_{k}\left[\sigma^{2}\left(\lambda_{n}+\beta_{m}\right)-a_{1}\right]\right)^{2} \\
& +h_{k}^{2}\left[\sigma^{2}\left(\lambda_{n}+\beta_{m}\right)-a_{1}\right]^{2} \mathbb{E} V(u(t)) \\
& +4 h_{k}^{4} a_{2}^{2}(\mathbb{E} V(u(t)))^{\frac{1}{2}}(\mathbb{E} V(u(t)))^{\frac{1}{2}} \\
& +4 h_{k}^{2} a_{2}^{2}(\mathbb{E} V(u(t)))^{\frac{1}{2}}(\mathbb{E} V(u(t)))^{\frac{1}{2}}
\end{aligned}
$$

thus

$$
\begin{aligned}
\mathbb{E} \| v(t) & -\hat{f}_{c}(u(t)) \|_{N}^{2} \\
\leq \quad & 4 h_{k}^{2}\left(\kappa+h_{k}\left[\sigma^{2}\left(\lambda_{n}+\beta_{m}\right)-a_{1}\right]\right)^{2} \mathbb{E} V(u(t)) \\
& +h_{K}^{2}\left[\sigma^{2}\left(\lambda_{n}+\beta_{m}\right)-a_{1}\right]^{2} \mathbb{E} V(u(t)) \\
& +4 h_{k}^{2} a_{2}^{2}\left(1+h_{k}^{2}\right) \mathbb{E} V(u(t)) \\
=\quad & 4 h_{k}^{2}\left(\kappa+h_{k}\left[\sigma^{2}\left(\lambda_{n}+\beta_{m}\right)-a_{1}\right]\right)^{2} \mathbb{E} V(u(t)) \\
& +4 h_{k}^{2}\left[\sigma^{2}\left(\lambda_{n}+\beta_{m}\right)-a_{1}\right]^{2} \mathbb{E} V(u(t)) \\
& +4 h_{k}^{2} a_{2}^{2}\left(1+h_{k}^{2}\right) \mathbb{E} V(u(t)) \\
\leq \quad & 4 h_{k}^{2}\left(\kappa+\sigma^{2}\left(\lambda_{n}+\beta_{m}\right)-a_{1}\right)^{2} \mathbb{E} V(u(t)) \\
& +4 h_{k}^{2}\left[\sigma^{2}\left(\lambda_{n}+\beta_{m}\right)-a_{1}\right]^{2} \mathbb{E} V(u(t)) \\
& +8 h_{k}^{2} a_{2}^{2} \mathbb{E} V(u(t)),
\end{aligned}
$$

for large $N$ and take the conditional expectation, then we get

$$
\begin{aligned}
& \mathbb{E}\left[\left\|v(t)-\hat{f}_{c}(u(t))\right\|_{N}^{2} \mid u(t)\right] \\
& \leq \quad 4\left[\kappa+\sigma^{2} N^{2} \pi^{2}\left(\frac{1}{l_{x}^{2}}+\frac{1}{l_{y}^{2}}\right)^{2}\right](1+V(u(t))) h_{k}^{2} \\
& \quad+4\left[\sigma^{4} N^{4} \pi^{4}\left(\frac{1}{l_{x}^{2}}+\frac{1}{l_{y}^{2}}\right)^{2}+2 a_{2}^{2}\right](1+V(u(t))) h_{k}^{2}
\end{aligned}
$$

thus

$$
\mathbb{E}\left[\left\|v(t)-\hat{f}_{c}(u(t))\right\|_{N}^{2} \mid u(t)\right] \leq K_{6} \tilde{V}(u(t)) h_{k}^{2}
$$


where

$$
K_{6}=4\left[\kappa+\sigma^{2} N^{2} \pi^{2}\left(\frac{1}{l_{x}^{2}}+\frac{1}{l_{y}^{2}}\right)^{2}+\sigma^{4} N^{4} \pi^{4}\left(\frac{1}{l_{x}^{2}}+\frac{1}{l_{y}^{2}}\right)^{2}+2 a_{2}^{2}\right] .
$$

Therefore,

$$
\begin{gathered}
\int_{t}^{t+h} \mathbb{E}\left[\left\|v(t)-\hat{f}_{c}(u(t))\right\|_{N}^{2} \mid u(t)\right] d s \\
\leq \quad K_{6} \tilde{V}(u(t)) h_{k}^{2} \int_{t}^{t+h} d s \\
\leq K_{6} \tilde{V}(u(t)) h_{k}^{3}
\end{gathered}
$$

and since $h_{k} \leq 1$, then

$$
\int_{t}^{t+h} \mathbb{E}\left[\left\|v(t)-\hat{f}_{c}(u(t))\right\|_{N}^{2} \mid u(t)\right] d s \leq K_{6} \tilde{V}(u(t)) h_{k}^{2}
$$

Form inequalities (20) and (21), we have

$$
\begin{array}{rl}
\int_{t}^{t+h} & \mathbb{E}\left[\left\|v(s)-\hat{f}_{c}(u(t))\right\|_{N}^{2} \mid u(t)\right] d s \\
\quad \leq \quad K_{5} \tilde{V}(u(t)) h_{k}^{2}+K_{6} \tilde{V}(u(t)) h_{k}^{2} \\
\quad \leq \quad K_{7} \tilde{V}(u(t)) h_{k}^{2}
\end{array}
$$

where $K_{7}=K_{5}+K_{6}$. Now, the second part of inequality (16) is

$$
\begin{aligned}
\mathbb{E} \| & \int_{t}^{t+h} \hat{g}_{c}(u(t)) d W(s) \|^{2} \\
& =\mathbb{E}\left\|\int_{t}^{t+h} \frac{h_{k}\left(b_{0}+b_{1}\|u(t)\|_{l^{2}}+b_{2}\|v(t)\|_{l^{2}}\right) \alpha^{2}}{F_{c}(u(t))} d W(s)\right\|_{N}^{2} \\
& =\mathbb{E}\left\|\frac{h_{k}\left(b_{0}+b_{1}\|u(t)\|_{l^{2}}+b_{2}\|v(t)\|_{l^{2}}\right) \alpha^{2}}{F_{c}(u(t))} \int_{t}^{t+h} d W(s)\right\|_{N}^{2} .
\end{aligned}
$$

Then

$$
\begin{aligned}
& \mathbb{E} \| \int_{t}^{t+h} \hat{g}_{c}(u(t)) d W(s)_{N}^{2} \\
& \leq \mathbb{E}\left[\left\|\frac{h_{k}\left(b_{0}+b_{1}\|u(t)\|+b_{2}\|v(t)\|\right) \alpha^{2}}{F_{c}(u(t))}\right\|_{N}^{2}\left\|\int_{t}^{t+h} d W(s)\right\|_{N}^{2}\right] \\
& \leq\left(\mathbb{E}\left\|\frac{h_{k}\left(b_{1}\|u(t)\|+b_{2}\|v(t)\|\right) \alpha^{2}}{F_{c}(u(t))}\right\|_{N}^{4}\right)^{\frac{1}{2}}\left(\mathbb{E}\left\|\int_{t}^{t+h} d W(s)\right\|_{N}^{4}\right)^{\frac{1}{2}}
\end{aligned}
$$

using Lemma 5.1, $F_{c}(u(t)) \geq 0$, and

$(a+b+c)^{4}=\left((a+b+c)^{2}\right)^{2} \leq\left(3\left(a^{2}+b^{2}+c^{2}\right)\right)^{2} \leq 27\left(a^{4}+b^{4}+c^{4}\right)$, then we find

$$
\begin{aligned}
& \mathbb{E}\left\|\int_{t}^{t+h} \hat{g}_{c}(u(t)) d W(s)\right\|_{N}^{2} \\
& \quad \leq \quad h_{k}^{2} \alpha^{4}\left(27\left[b_{0}^{4}+b_{1}^{4} \mathbb{E}\|u(t)\|^{4}+b_{2}^{4} \mathbb{E}\|v(t)\|^{4}\right]\right)^{\frac{1}{2}}\left(3 h_{k}^{2}\right)^{\frac{1}{2}} \\
& \quad \leq 9 \alpha^{4}\left(b_{0}^{4}+b_{1}^{4} \mathbb{E} V(u(t))+b_{2}^{4} \mathbb{E} V^{2}(u(t))\right)^{\frac{1}{2}} h_{k}^{3} .
\end{aligned}
$$

Hence, by redoing above steps for the conditional expectation, we arrive at

$$
\begin{aligned}
\mathbb{E}\left[\| \int_{t}^{t+h} \hat{g}_{c}(u(t))\right. & \left.d W(s) \|_{N}^{2} \mid \mathcal{F}_{t}\right] \\
& \leq 9 \alpha^{4}\left(b_{0}^{4}+b_{1}^{4} V(u(t))+b_{2}^{4} V^{2}(u(t))\right)^{\frac{1}{2}} h_{k}^{3} \\
& \leq 9 \alpha^{4}\left(b_{0}^{2}+b_{1}^{2} \sqrt{V(u(t))}+b_{2}^{2} V(u(t))\right) h_{k}^{3} \\
& \leq 9 \alpha^{4}\left(b_{0}^{2}+\frac{b_{1}^{4}}{2}+\frac{V(u(t))}{2}+b_{2}^{2} V(u(t))\right) h_{k}^{3},
\end{aligned}
$$

because we know from the Young's inequality that if $p=2$ and $q=$ 2 , that $a \cdot b \leq \frac{a^{2}}{2}+\frac{b^{2}}{2}$, then

$$
\begin{aligned}
& \mathbb{E}\left[\| \int_{t}^{t+h} \hat{g}_{c}(u(t))\right.\left.d W(s) \|_{N}^{2} \mid \mathcal{F}_{t}\right] \\
& \leq 9 \alpha^{4}\left(b_{0}^{2}+\frac{b_{1}^{4}}{2}+\left(\frac{1}{2}+b_{2}^{2}\right) V(u(t))\right) h_{k}^{3}
\end{aligned}
$$

and therefore

$$
\begin{aligned}
\mathbb{E}\left[\| \int_{t}^{t+h}\right. & \left.\hat{g}_{c}(u(t)) d W(s) \|_{N}^{2} \mid \mathcal{F}_{t}\right] \\
& \leq 9 \alpha^{4} \max \left\{b_{0}^{2}+\frac{b_{1}^{4}}{2}+\frac{1}{2}+b_{2}^{2}, \frac{1}{2}+b_{2}^{2}\right\} V(u(t)) h_{k}^{2} .
\end{aligned}
$$

Thus

$$
\begin{aligned}
\mathbb{E}\left\|\int_{t}^{t+h} \hat{g}_{c}(u(t)) d W(s)\right\|_{N}^{2} & \leq K_{8} V(u(t)) h_{k}^{2} \\
& \leq k_{8}(1+V(u(t))) h_{k}^{2} \\
& \left.\leq k_{8} \tilde{V}(u(t))\right) h_{k}^{2} .
\end{aligned}
$$

where

$$
K_{8}=9 \alpha^{4} \max \left\{b_{0}^{2}+\frac{b_{1}^{4}}{2}+\frac{1}{2}+b_{2}^{2}, \frac{1}{2}+b_{2}^{2}\right\} .
$$

Thus inequality (16) equivalent to

$$
\begin{aligned}
\mathbb{E}[\| c(t+h) & \left.-\hat{c}(t+h) \|_{N}^{2} \mid \mathcal{F}_{t}\right] \\
& \leq 2\left(K_{7} \tilde{V}(u(t)) h_{k}^{2}+2 K_{8} \tilde{V}(u(t)) h_{k}^{2}\right) \\
& \leq K_{c_{2}}^{c} \tilde{V}(u(t)) h_{k}^{2}
\end{aligned}
$$

where $K_{c_{2}}^{c}=2\left(K_{7}+K_{8}\right)$, which is implies that

$$
\left(\mathbb{E}\left[\|c(t+h)-\hat{c}(t+h)\|_{N}^{2} \mid \mathcal{F}_{t}\right]\right)^{\frac{1}{2}} \leq\left(K_{c_{2}}^{c}\right)^{\frac{1}{2}}(\tilde{V}(u(t)))^{\frac{1}{2}} h_{k}
$$

2) To prove inequality (15), from [10] we know that,

$$
\begin{aligned}
v(t+h)-\hat{v}(t+h)= & \int_{t}^{t+h}\left[f_{v}(u(s))-\hat{f}_{v}(u(t))\right] d s \\
& +\int_{t}^{t+h}\left[g_{v}(u(s))-\hat{g}_{v}(u(t))\right] d W(s)
\end{aligned}
$$

thus

$$
\begin{aligned}
v(t+h) & -\hat{v}(t+h) \|_{N}^{2} \\
& \leq 2\left\|\int_{t}^{t+h}\left[f_{v}(u(s))-\hat{f}_{v}(u(t))\right] d s\right\|_{N}^{2} \\
& +2 \int_{t}^{t+h}\left[g_{v}(u(s))-\hat{g}_{v}(u(t))\right] d W(s) \|_{N}^{2}
\end{aligned}
$$

and we know that

$$
\begin{aligned}
f_{v}(u(s))-\hat{f}_{v}(u(t))= & f_{v}(u(s))-f_{v}(u(t))+f_{v}(u(t)) \\
& -f_{v}(\hat{u}(t))+f_{v}(\hat{u}(t))-\hat{f}_{v}(\hat{u}(t))
\end{aligned}
$$

and, by using the local property that $u(t)=\hat{u}(t)$, hence

$$
\begin{aligned}
\left\|f_{v}(u(s))-\hat{f}_{v}(u(t))\right\|_{N}^{2} & \leq 2\left\|f_{v}(u(s))-f_{v}(u(t))\right\|_{N}^{2} \\
& +2\left\|f_{v}(\hat{u}(t))-\hat{f}_{v}(\hat{u}(t))\right\|_{N}^{2}
\end{aligned}
$$

where

$$
\begin{aligned}
f_{v}(u(s))-f_{v}(u(t))= & {\left[\sigma^{2}\left(\lambda_{n}+\beta_{m}\right)-a_{1}\right](c(t)-c(s)) } \\
& +a_{2}\left(\|u(t)\|_{N}^{2} c(t)-\|u(s)\|_{N}^{2} c(s)\right) \\
& +\kappa(v(t)-v(s))
\end{aligned}
$$


thus

$$
\begin{aligned}
\left\|f_{v}(u(s))-f_{v}(u(t))\right\|_{N}^{2} & \leq 3\left(\sigma^{2}\left(\lambda_{n}+\beta_{m}\right)-a_{1}\right){ }^{2}\|c(t)-c(s)\|_{N}^{2} \\
& +3 a_{2}^{2}\|\| u(t)\left\|_{N}^{2} c(t)-\right\| u(s)\left\|_{N}^{2} c(s)\right\|_{N}^{2} \\
& +3 \kappa^{2}\|v(t)-v(s)\|_{N}^{2} .
\end{aligned}
$$

Also, from [10], we have

$$
\begin{aligned}
\|\| u(t) \|_{N}^{2} c(t) & -\|u(s)\|_{N}^{2} c(s) \|_{N} \\
& \leq 3\|c(\gamma)\|_{N}^{2}\|v(\gamma)\|_{N}\|c(t)-c(s)\|_{N}
\end{aligned}
$$

hence

$$
\begin{aligned}
& \mathbb{E}\|\| u(t)\left\|_{N}^{2} c(t)-\right\| u(s)\left\|_{N}^{2} c(s)\right\|_{N} \\
& \quad \leq 9 \mathbb{E}\left(\|c(\gamma)\|_{N}^{4}\|v(\gamma)\|_{N}^{2}\|c(t)-c(s)\|_{N}^{2}\right) \\
& \quad \leq 9\left[\mathbb{E}\left(\|c(\gamma)\|_{N}^{8}\|v(\gamma)\|_{N}^{4}\right)\right]^{\frac{1}{2}}\left[\mathbb{E}\|c(t)-c(s)\|_{N}^{4}\right]^{\frac{1}{2}} \\
& \quad \leq 9\left[\mathbb{E}\|c(\gamma)\|_{N}^{16}\right]^{\frac{1}{2}}\left[\mathbb{E}\|v(\gamma)\|_{N}^{8}\right]^{\frac{1}{2}}\left[\mathbb{E}\|c(t)-c(s)\|_{N}^{4}\right]^{\frac{1}{2}} \\
& \quad \leq \frac{36}{a_{2}^{2}}\left[\mathbb{E} V^{4}(u(t))\right]^{\frac{1}{2}}\left[\mathbb{E} V^{4}(u(t))\right]^{\frac{1}{2}}\left[\mathbb{E}\|c(t)-c(s)\|_{N}^{4}\right]^{\frac{1}{2}}
\end{aligned}
$$

therefore

$$
\begin{aligned}
\mathbb{E}\|\| u(t)\left\|_{N}^{2} c(t)-\right\| u(s)\left\|_{N}^{2} c(s)\right\|_{N} \leq \\
\frac{36}{a_{2}^{2}} \mathbb{E} V^{4}(u(t))\left[\mathbb{E}\|c(t)-c(s)\|_{N}^{4}\right]^{\frac{1}{2}}
\end{aligned}
$$

but we can find that

$$
\begin{aligned}
\mathbb{E}\|c(t)-c(s)\|_{N}^{4} & \leq \mathbb{E}\left(\int_{t}^{s}[\mathbb{E} V(u(r))]^{\frac{1}{2}} d r\right)^{4} \\
& \leq \int_{t}^{s}[\mathbb{E} V(u(r))]^{2} d r(s-t)^{2} \\
& \leq[\mathbb{E} V(u(t))]^{2}(s-t)^{2} \int_{t}^{s} d r
\end{aligned}
$$

and so

$$
\mathbb{E}\|c(t)-c(s)\|_{N}^{4} \leq[\mathbb{E} V(u(t))]^{2}(s-t)^{3} .
$$

Substituting inequality (27) in inequality (26), we find that

$$
\begin{aligned}
\mathbb{E}\|\| u(t) \|_{N}^{2} c(t) & -\|u(s)\|_{N}^{2} c(s) \|_{N} \\
& \leq \frac{36}{a_{2}^{2}} \mathbb{E} V^{4}(u(t))[\mathbb{E} V(u(t))]^{2}(s-t)^{3}
\end{aligned}
$$

and we know that

$$
\mathbb{E}\|\| c(t)-c(s)\left\|_{N}^{2} c(s)\right\|_{N} \leq \mathbb{E} V(u(t))(s-t)^{2} .
$$

Also from Theorem 2 part 1), we know that

$$
\int_{t}^{t+h} \mathbb{E}\|v(t)-v(s)\|_{N}^{2} d s \leq K_{5} \tilde{V}(u(t)) h_{k}^{2} .
$$

Therefore, by using inequalities (28), (29), and (30), we find that

$$
\begin{aligned}
\int_{t}^{t+h} \mathbb{E} \| f_{v} & (u(s))-f_{v}(u(t)) \|_{N}^{2} d s \\
\leq & 3\left[\sigma^{2}\left(\lambda_{n}+\beta_{m}\right)-a_{1}\right]^{2} \int_{t}^{t+h} \mathbb{E}\|c(t)-c(s)\|_{N}^{2} d s \\
& +3 a_{2}^{2} \int_{t}^{t+h} \mathbb{E}\|\| u(t)\left\|_{N}^{2} c(t)-\right\| u(s)\left\|_{N}^{2} c(s)\right\|_{N}^{2} d s \\
& +3 \kappa^{2} \int_{t}^{t+h} \mathbb{E}\|v(t)-v(s)\|_{N}^{2} d s,
\end{aligned}
$$

hence

$$
\begin{aligned}
\int_{t}^{t+h} \mathbb{E}\left[\left\|f_{v}(u(s))-f_{v}(u(t))\right\|_{N}^{2} \mid u(t)\right] d s \\
\leq \quad 3\left[\sigma^{2}\left(\lambda_{n}+\beta_{m}\right)-a_{1}\right]^{2} \int_{t}^{t+h} V(u(t))(s-t)^{2} d s \\
+108 \int_{t}^{t+h} V^{4}(u(t)) \mathbb{E} V(u(t))|s-t|^{3} d s \\
+3 K_{5} \kappa^{2} \tilde{V}(u(t)) h_{k}^{2} \\
\leq \quad\left[\sigma^{2}\left(\lambda_{n}+\beta_{m}\right)-a_{1}\right]^{2} V(u(t)) h_{k}^{3} \\
+\frac{108}{4} V^{4}(u(t)) V(u(t)) h_{k}^{4}+3 K_{5} \kappa \tilde{V}(u(t)) h_{k}^{2} \\
\leq \quad\left[\sigma^{2}\left(\lambda_{n}+\beta_{m}\right)-a_{1}\right]^{2} V(u(t)) h_{k}^{2} \\
+27 V^{4}(u(t)) V(u(t)) h_{k}^{2}+3 K_{5} \kappa \tilde{V}(u(t)) h_{k}^{2} .
\end{aligned}
$$

Thus, for large $N$, we have

$$
\begin{aligned}
\int_{t}^{t+h} \mathbb{E}\left[\left\|f_{v}(u(s))-f_{v}(u(t))\right\|_{N}^{2} \mid u(t)\right] d s \\
\leq \quad \sigma^{4} N^{4} \pi^{4}\left(\frac{1}{l_{x}^{2}}+\frac{1}{l_{y}^{2}}\right)^{2} V(u(t)) h_{k}^{2} \\
\quad+27 V^{4}(u(t)) V(u(t)) h_{k}^{2}+3 K_{5} \kappa \tilde{V}(u(t)) h_{k}^{2} \\
\leq \quad \sigma^{4} N^{4} \pi^{4}\left(\frac{1}{l_{x}^{2}}+\frac{1}{l_{y}^{2}}\right)^{2}(1+V(u(t))) h_{k}^{2} \\
\quad+27 V^{4}(u(t))(1+V(u(t))) h_{k}^{2}+3 K_{5} \kappa \tilde{V}(u(t)) h_{k}^{2}
\end{aligned}
$$

therefore

where 
The second part of inequality (25) is,

$$
\begin{aligned}
\left\|f_{v}(\hat{u}(t))-\hat{f}_{v}(\hat{u}(t))\right\|_{N}^{2} & \\
\leq & h_{k}^{2}\left[\left\|\frac{\kappa\left[\sigma^{2}\left(\lambda_{n}+\beta_{m}\right)-a_{1}+a_{2}\|\hat{u}(t)\|_{N}^{2}\right] \hat{c}(t)}{F(\hat{u}(t))}\right\|_{N}^{2}\right. \\
& +\left\|\frac{h_{k}\left[\sigma^{2}\left(\lambda_{n}+\beta_{m}\right)-a_{1}+a_{2}\|\hat{u}(t)\|_{N}^{2}\right]^{2} \hat{c}(t)}{F(\hat{u}(t))}\right\|_{N}^{2}+\left\|\frac{\kappa^{2} \hat{v}(t)}{F(\hat{u}(t))}\right\|_{N}^{2} \\
& \left.+\left\|\frac{h_{k} \kappa\left[\sigma^{2}\left(\lambda_{n}+\beta_{m}\right)-a_{1}+a_{2}\|\hat{u}(t)\|_{N}^{2}\right] \hat{v}(t)}{F(\hat{u}(t))}\right\|_{N}^{2}\right] \\
\leq \quad & 4 h_{k}^{2}\left[\kappa^{2}\left[\sigma^{2}\left(\lambda_{n}+\beta_{m}\right)-a_{1}+a_{2}\|\hat{u}(t)\|_{N}^{2}\right]^{2}\left\|\frac{\hat{c}(t)}{F(\hat{u}(t))}\right\|_{N}^{2}\right. \\
& +h_{k}^{2}\left[\sigma^{2}\left(\lambda_{n}+\beta_{m}\right)-a_{1}+a_{2}\|\hat{u}(t)\|_{N}^{2}\right]^{4}\left\|\frac{\hat{c}(t)}{F(\hat{u}(t))}\right\|_{N}^{2} \\
& +\kappa^{4}\left\|\frac{\hat{v}(t)}{F(\hat{u}(t))}\right\|_{N}^{2} \\
& \left.+h_{k}^{2} \kappa^{2}\left[\sigma^{2}\left(\lambda_{n}+\beta_{m}\right)-a_{1}+a_{2}\|\hat{u}(t)\|_{N}^{2}\right]^{2}\left\|\frac{\hat{v}(t)}{F(\hat{u}(t))}\right\|_{N}^{2}\right] \\
\leq \quad & h_{k}^{2}\left[\kappa ^ { 2 } \left(\left[\sigma^{2}\left(\lambda_{n}+\beta_{m}\right)-a_{1}+a_{2}\|\hat{u}(t)\|_{N}^{2}\right]^{2}\left\|\frac{\hat{c}(t)}{F(\hat{u}(t))}\right\|_{N}^{2}\right.\right. \\
& \left.+\kappa^{2}\left\|\frac{\hat{v}(t)}{F(\hat{u}(t))}\right\|_{N}^{2}\right) \\
& +h_{k}^{2}\left[\sigma^{2}\left(\lambda_{n}+\beta_{m}\right)-a_{1}+a_{2}\|\hat{u}(t)\|_{N}^{2}\right]^{2}\left(\left[\sigma^{2}\left(\lambda_{n}+\beta_{m}\right)-a_{1}\right.\right. \\
& \left.\left.\left.+a_{2}\|\hat{u}(t)\|_{N}^{2}\right]^{2}\left\|\frac{\hat{c}(t)}{F(\hat{u}(t))}\right\|_{N}^{2}+\kappa^{2}\left\|\frac{\hat{v}(t)}{F(\hat{u}(t))}\right\|_{N}^{2}\right)\right]
\end{aligned}
$$

hence

$$
\begin{aligned}
\left\|f_{v}(\hat{u}(t))-\hat{f}_{v}(\hat{u}(t))\right\|_{N}^{2} \\
\leq \quad 4 h_{k}^{2}\left[\left(\kappa^{2}+h_{k}^{2}\left[\sigma^{2}\left(\lambda_{n}+\beta_{m}\right)-a_{1}+a_{2}\|\hat{u}(t)\|_{N}^{2}\right]^{2}\right)\right. \\
\quad\left(\left[\sigma^{2}\left(\lambda_{n}+\beta_{m}\right)-a_{1}+a_{2} \| \text { hatu }(t) \|_{N}^{2}\right]^{2}\left\|\frac{\hat{c}(t)}{F(\hat{u}(t))}\right\|_{N}^{2}\right. \\
\left.\left.\quad+\kappa^{2}\left\|\frac{\hat{v}(t)}{F(\hat{u}(t))}\right\|_{N}^{2}\right)\right]
\end{aligned}
$$

using $F(\hat{u}(t)) \geq 1$, and pulling the expectation over the last identity, then we find

$$
\begin{aligned}
& \mathbb{E}\left\|f_{v}(\hat{u}(t))-\hat{f}_{v}(\hat{u}(t))\right\|_{N}^{2} \\
& \leq 4 h_{k}^{2} \mathbb{E}\left[( \kappa ^ { 2 } + h _ { k } ^ { 2 } [ \sigma ^ { 2 } ( \lambda _ { n } + \beta _ { m } ) - a _ { 1 } + a _ { 2 } \| \hat { u } ( t ) \| _ { N } ^ { 2 } ] ^ { 2 } ) \left(\left[\sigma^{2}\left(\lambda_{n}+\beta_{m}\right)\right.\right.\right. \\
& \left.\left.\left.\quad-a_{1}+a_{2}\|\hat{u}(t)\|_{N}^{2}\right]^{2}\left\|\frac{\hat{c}(t)}{F(\hat{u}(t))}\right\|_{N}^{2}+\kappa^{2}\left\|\frac{\hat{v}(t)}{F(\hat{u}(t))}\right\|_{N}^{2}\right)\right]
\end{aligned}
$$

therefore,

$$
\begin{aligned}
\mathbb{E}\left\|f_{v}(\hat{u}(t))-\hat{f}_{v}(\hat{u}(t))\right\|_{N}^{2} \\
\leq 4 h_{k}^{2} \mathbb{E}\left[\left(\kappa^{2}+\left[\sigma^{2}\left(\lambda_{n}+\beta_{m}\right)-a_{1}+a_{2}\|\hat{u}(t)\|_{N}^{2}\right]^{2}\right)\right. \\
\quad\left(\left[\sigma^{2}\left(\lambda_{n}+\beta_{m}\right)-a_{1}+a_{2}\|\hat{u}(t)\|_{N}^{2}\right]^{2}\left\|\frac{\hat{c}(t)}{F(\hat{u}(t))}\right\|_{N}^{2}\right. \\
\left.\left.\quad+\kappa^{2}\left\|\frac{\hat{v}(t)}{F(\hat{u}(t))}\right\|_{N}^{2}\right)\right]
\end{aligned}
$$

hence

$$
\begin{aligned}
\mathbb{E}\left\|f_{v}(\hat{u}(t))-\hat{f}_{v}(\hat{u}(t))\right\|_{N}^{2} \\
\leq 4 h_{k}^{2}\left(\mathbb{E}\left[\kappa^{2}+\left[\sigma^{2}\left(\lambda_{n}+\beta_{m}\right)-a_{1}+a_{2}\|\hat{u}(t)\|_{N}^{2}\right]^{2}\right]^{2}\right)^{\frac{1}{2}} \\
\quad\left[\mathbb { E } \left(\left[\sigma^{2}\left(\lambda_{n}+\beta_{m}\right)-a_{1}+a_{2}\|\hat{u}(t)\|_{N}^{2}\right]^{2}\left\|\frac{\hat{c}(t)}{F(\hat{u}(t))}\right\|_{N}^{2}\right.\right. \\
\left.\left.+\kappa^{2}\left\|\frac{\hat{v}(t)}{F(\hat{u}(t))}\right\|_{N}^{2}\right)^{2}\right]^{\frac{1}{2}}
\end{aligned}
$$

for large $N$, we have

$$
\begin{aligned}
\mathbb{E}\left\|f_{v}(\hat{u}(t))-\hat{f}_{v}(\hat{u}(t))\right\|_{N}^{2} & \\
\leq & 4 h_{k}^{2}\left(\mathbb{E}\left[\kappa^{2}+\left[\sigma^{2} N^{2} \pi^{2}\left(\frac{1}{l_{x}^{2}}+\frac{1}{l_{y}^{2}}\right)^{2}+a_{2}\|\hat{u}(t)\|_{N}^{2}\right]^{2}\right]^{2}\right)^{\frac{1}{2}} \\
& {\left[\mathbb { E } \left(\left[\sigma^{2} N^{2} \pi^{2}\left(\frac{1}{l_{x}^{2}}+\frac{1}{l_{y}^{2}}\right)^{2}+a_{2}\|\hat{u}(t)\|_{N}^{2}\right]^{2}\left\|\frac{\hat{c}(t)}{F(\hat{u}(t))}\right\|_{N}^{2}\right.\right.} \\
& \left.\left.+\kappa^{2}\left\|\frac{\hat{v}(t)}{F(\hat{u}(t))}\right\|_{N}^{2}\right)^{2}\right]^{\frac{1}{2}} .
\end{aligned}
$$

First, I will calculate the first part of the right side of inequality (32) which is

$$
\begin{aligned}
(\mathbb{E} & {\left.\left[\kappa^{2}+h_{k}^{2}\left[\sigma^{2} N^{2} \pi^{2}\left(\frac{1}{l_{x}^{2}}+\frac{1}{l_{y}^{2}}\right)^{2}+a_{2}\|\hat{u}(t)\|_{N}^{2}\right]^{2}\right]^{2}\right)^{\frac{1}{2}} } \\
& \leq\left(\mathbb{E}\left[2 \kappa^{4}+2\left[\sigma^{2} N^{2} \pi^{2}\left(\frac{1}{l_{x}^{2}}+\frac{1}{l_{y}^{2}}\right)^{2}+a_{2}\|\hat{u}(t)\|_{N}^{2}\right]^{4}\right]\right)^{\frac{1}{2}} \\
& \leq\left(\mathbb{E}\left[2 \kappa^{4}+2\left[4\left(\sigma^{2} N^{2} \pi^{2}\left(\frac{1}{l_{x}^{2}}+\frac{1}{l_{y}^{2}}\right)^{2}\right)^{4}+4 a_{2}^{4}\|\hat{u}(t)\|_{N}^{8}\right]\right]\right)^{\frac{1}{2}}
\end{aligned}
$$

hence

$$
\begin{aligned}
(\mathbb{E} & {\left.\left[\kappa^{2}+h_{k}^{2}\left[\sigma^{2} N^{2} \pi^{2}\left(\frac{1}{l_{x}^{2}}+\frac{1}{l_{y}^{2}}\right)^{2}+a_{2}\|\hat{u}(t)\|_{N}^{2}\right]^{2}\right]^{2}\right)^{\frac{1}{2}} } \\
& \leq\left(2 \kappa^{4}+2\left[4\left(\sigma^{2} N^{2} \pi^{2}\left(\frac{1}{l_{x}^{2}}+\frac{1}{l_{y}^{2}}\right)^{2}\right)^{4}+4 a_{2}^{4} \mathbb{E}\|\hat{u}(t)\|_{N}^{8}\right]\right)^{\frac{1}{2}} \\
& \leq\left(2 \kappa^{4}+8\left[\left(\sigma^{2} N^{2} \pi^{2}\left(\frac{1}{l_{x}^{2}}+\frac{1}{l_{y}^{2}}\right)^{2}\right)^{4}+a_{2}^{4} \mathbb{E} V^{2}(u(t))\right]\right)^{\frac{1}{2}} \\
& =\left(2 \kappa^{4}+8\left(\sigma^{2} N^{2} \pi^{2}\left(\frac{1}{l_{x}^{2}}+\frac{1}{l_{y}^{2}}\right)^{2}\right)^{4}+8 a_{2}^{4} \mathbb{E} V^{2}(u(t))\right)^{\frac{1}{2}} \\
& \leq \sqrt{2}\left(\kappa^{2}+2\left(\sigma^{2} N^{2} \pi^{2}\left(\frac{1}{l_{x}^{2}}+\frac{1}{l_{y}^{2}}\right)^{2}\right)^{2}+2 a_{2}^{2}\left[\mathbb{E} V^{2}(u(t))\right]^{\frac{1}{2}}\right) \\
& \leq \sqrt{2}\left(\kappa^{2}+2 \sigma^{4} N^{4} \pi^{4}\left(\frac{1}{l_{x}^{2}}+\frac{1}{l_{y}^{2}}\right)^{4}+2 a_{2}^{2}\left[\mathbb{E} V^{2}(u(t))\right]^{\frac{1}{2}}\right) .
\end{aligned}
$$

Now, the second part of the right side of inequality (32) is

$$
\begin{aligned}
& \mathbb{E}\left(\left[\sigma^{2} N^{2} \pi^{2}\left(\frac{1}{l_{x}^{2}}+\frac{1}{l_{y}^{2}}\right)^{2}+a_{2}\|\hat{u}(t)\|_{N}^{2}\right]^{2}\left\|\frac{\hat{c}(t)}{F(\hat{u}(t))}\right\|_{N}^{2}+\kappa^{2}\left\|\frac{\hat{v}(t)}{F(\hat{u}(t))}\right\|_{N}^{2}\right)^{2} \\
& \leq \quad 2 \mathbb{E}\left(\left[\sigma^{2} N^{2} \pi^{2}\left(\frac{1}{l_{x}^{2}}+\frac{1}{l_{y}^{2}}\right)^{2}+a_{2}\|\hat{u}(t)\|_{N}^{2}\right]^{4}\left\|\frac{\hat{c}(t)}{F(\hat{u}(t))}\right\|_{N}^{4}\right) \\
& \quad+2 \kappa^{2} \mathbb{E}\left\|\frac{\hat{v}(t)}{F(\hat{u}(t))}\right\|_{N}^{2} \\
& \leq \quad 2\left(\mathbb{E}\left[\sigma^{2} N^{2} \pi^{2}\left(\frac{1}{l_{x}^{2}}+\frac{1}{l_{y}^{2}}\right)^{2}+a_{2}\|\hat{u}(t)\|_{N}^{2}\right]^{8}\right)^{\frac{1}{2}}\left(\mathbb{E}\left\|\frac{\hat{c}(t)}{F(\hat{u}(t))}\right\|_{N}^{8}\right)^{\frac{1}{2}} \\
& \quad+2 \kappa^{2} \mathbb{E}\left\|\frac{\hat{v}(t)}{F(\hat{u}(t))}\right\|_{N}^{2} \\
& \leq 2\left(16 \sigma^{16} N^{16} \pi^{16}\left(\frac{1}{l_{x}^{2}}+\frac{1}{l_{y}^{2}}\right)^{16}+a_{2}^{8} \mathbb{E}\|\hat{u}(t)\|_{N}^{16}\right)^{\frac{1}{2}} \\
&\left(\mathbb{E}\left\|\frac{\hat{c}(t)}{F(\hat{u}(t))}\right\|_{N}^{8}\right)^{\frac{1}{2}}+2 \kappa^{2} \mathbb{E}\left\|\frac{\hat{v}(t)}{F(\hat{u}(t))}\right\|_{N}^{2}
\end{aligned}
$$


but $F(\hat{u}(t)) \geq 1$, then

$$
\begin{aligned}
\mathbb{E}\left(\left[\sigma^{2}\right.\right. & \left.\left.N^{2} \pi^{2}\left(\frac{1}{l_{x}^{2}}+\frac{1}{l_{y}^{2}}\right)^{2}+a_{2}\|\hat{u}(t)\|_{N}^{2}\right]^{2}\|\hat{c}(t)\|_{N}^{2}+\kappa^{2}\|\hat{v}(t)\|_{N}^{2}\right)^{2} \\
\leq & 2\left(16 \sigma^{16} N^{16} \pi^{16}\left(\frac{1}{l_{x}^{2}}+\frac{1}{l_{y}^{2}}\right)^{16}+a_{2}^{8} \mathbb{E}\|\hat{u}(t)\|_{N}^{16}\right)^{\frac{1}{2}}\left(\mathbb{E}\|\hat{c}(t)\|_{N}^{8}\right)^{\frac{1}{2}} \\
& +2 \kappa^{2} \mathbb{E}\|\hat{v}(t)\|_{N}^{2} \\
\leq & 2\left(4 \sigma^{8} N^{8} \pi^{8}\left(\frac{1}{l_{x}^{2}}+\frac{1}{l_{y}^{2}}\right)^{8}+a_{2}^{4} \mathbb{E}\|\hat{u}(t)\|_{N}^{8}\right)\left(\mathbb{E}\|\hat{c}(t)\|_{N}^{8}\right)^{\frac{1}{2}} \\
& +2 \kappa^{2} \mathbb{E}\|\hat{v}(t)\|_{N}^{2},
\end{aligned}
$$

hence

$\left[\mathbb{E}\left(\left[\sigma^{2} N^{2} \pi^{2}\left(\frac{1}{l_{x}^{2}}+\frac{1}{l_{y}^{2}}\right)^{2}+a_{2}\|\hat{u}(t)\|_{N}^{2}\right]^{2}\|\hat{c}(t)\|_{N}^{2}+\kappa^{2}\|\hat{v}(t)\|_{N}^{2}\right)^{2}\right]^{\frac{1}{2}}$ hence

$\leq\left[2\left(4 \sigma^{8} N^{8} \pi^{8}\left(\frac{1}{l_{x}^{2}}+\frac{1}{l_{y}^{2}}\right)^{8}+a_{2}^{4} \mathbb{E}\|\hat{u}(t)\|_{N}^{8}\right)\left(\mathbb{E}\|\hat{c}(t)\|_{N}^{8}\right)^{\frac{1}{2}}+2 \kappa^{2} \mathbb{E}\|\hat{v}(t)\|_{N}^{2}\right]^{\frac{1}{2}}$

$\leq \sqrt{2}\left(4 \sigma^{8} N^{8} \pi^{8}\left(\frac{1}{l_{x}^{2}}+\frac{1}{l_{y}^{2}}\right)^{8}+a_{2}^{4} \mathbb{E}\|\hat{u}(t)\|_{N}^{8}\right)^{\frac{1}{2}}\left(\mathbb{E}\|\hat{c}(t)\|_{N}^{8}\right)^{\frac{1}{4}}$

$+\sqrt{2} \kappa\left(\mathbb{E}\|\hat{v}(t)\|_{N}^{2}\right)^{\frac{1}{2}}$

$\leq \sqrt{2}\left(2 \sigma^{4} N^{4} \pi^{4}\left(\frac{1}{l_{x}^{2}}+\frac{1}{l_{y}^{2}}\right)^{4}+a_{2}^{2}\left(\mathbb{E}\|\hat{u}(t)\|_{N}^{8}\right)^{\frac{1}{2}}\right)\left(\mathbb{E}\|\hat{c}(t)\|_{N}^{8}\right)^{\frac{1}{4}}$

$+\sqrt{2} \kappa\left(\mathbb{E}\|\hat{v}(t)\|_{N}^{2}\right)^{\frac{1}{2}}$

$\leq \sqrt{2}\left(2 \sigma^{4} N^{4} \pi^{4}\left(\frac{1}{l_{x}^{2}}+\frac{1}{l_{y}^{2}}\right)^{4}+2 a_{2}\left(\mathbb{E} V^{2}(u(t))\right)^{\frac{1}{2}}\right)$

$\left(\frac{2}{a_{2}} \mathbb{E} V^{8}(u(t))\right)^{\frac{1}{4}}+\sqrt{2} \kappa(\mathbb{E} V(u(t)))^{\frac{1}{2}}$.

Thus inequality (32) equivalent to

$$
\begin{aligned}
& \mathbb{E}\left\|f_{v}(\hat{u}(t))-\hat{f}_{v}(\hat{u}(t))\right\|_{N}^{2} \\
& \leq \quad 4 h_{k}^{2} \cdot \sqrt{2}\left(\kappa^{2}+2 \sigma^{4} N^{4} \pi^{4}\left(\frac{1}{l_{x}^{2}}+\frac{1}{l_{y}^{2}}\right)^{4}+2 a_{2}^{2}\left[\mathbb{E} V^{2}(u(t))\right]^{\frac{1}{2}}\right) \\
& {\left[\sqrt{2}\left(2 \sigma^{4} N^{4} \pi^{4}\left(\frac{1}{l_{x}^{2}}+\frac{1}{l_{y}^{2}}\right)^{4}+2 a_{2}\left(\mathbb{E} V^{2}(u(t))\right)^{\frac{1}{2}}\right)\right.} \\
&\left.\left(\frac{2}{a_{2}} \mathbb{E} V^{8}(u(t))\right)^{\frac{1}{4}}+\sqrt{2} \kappa(\mathbb{E} V(u(t)))^{\frac{1}{2}}\right] .
\end{aligned}
$$

Pulling the conditional expectation over the last identity, we find that

$$
\begin{gathered}
\mathbb{E}\left[\left\|f_{v}(\hat{u}(t))-\hat{f}_{v}(\hat{u}(t))\right\|_{N}^{2} \mid u(t)\right] \\
\leq 8 h_{k}^{2}\left(\kappa^{2}+2 \sigma^{4} N^{4} \pi^{4}\left(\frac{1}{l_{x}^{2}}+\frac{1}{l_{y}^{2}}\right)^{4}+2 a_{2}^{2}\left[V^{2}(u(t))\right]^{\frac{1}{2}}\right) \\
{\left[\left(2 \sigma^{4} N^{4} \pi^{4}\left(\frac{1}{l_{x}^{2}}+\frac{1}{l_{y}^{2}}\right)^{4}+2 a_{2}\left(V^{2}(u(t))\right)^{\frac{1}{2}}\right)\right.} \\
\left.\left(\frac{2}{a_{2}} V^{8}(u(t))\right)^{\frac{1}{4}}+\kappa(V(u(t)))^{\frac{1}{2}}\right] .
\end{gathered}
$$

Thus

$$
\begin{aligned}
\mathbb{E}[ & \left.\left\|f_{v}(\hat{u}(t))-\hat{f}_{v}(\hat{u}(t))\right\|_{N}^{2} \mid u(t)\right] \\
\leq \quad & 8 h_{k}^{2}\left(\kappa^{2}+2 \sigma^{4} N^{4} \pi^{4}\left(\frac{1}{l_{x}^{2}}+\frac{1}{l_{y}^{2}}\right)^{4}+2 a_{2}^{2} V(u(t))\right) \\
& {\left[\left(2 \sigma^{4} N^{4} \pi^{4}\left(\frac{1}{l_{x}^{2}}+\frac{1}{l_{y}^{2}}\right)^{4}+2 a_{2} V(u(t))\right)\right.} \\
& \left.\left(\frac{2}{a_{2}}\right)^{\frac{1}{4}} V^{2}(u(t))+\kappa V^{\frac{1}{2}}(u(t))\right] \\
\leq & 8 h_{k}^{2} \cdot\left(\kappa^{2}+2 \sigma^{4} N^{4} \pi^{4}\left(\frac{1}{l_{x}^{2}}+\frac{1}{l_{y}^{2}}\right)^{4}+2 a_{2}^{2} V(u(t))\right) \\
& {\left[\left(2 \sigma^{4} N^{4} \pi^{4}\left(\frac{1}{l_{x}^{2}}+\frac{1}{l_{y}^{2}}\right)^{4}+2 a_{2} V(u(t))\right)\right.} \\
& \left.\left(\frac{2}{a_{2}}\right)^{\frac{1}{4}} V^{\frac{3}{2}}(u(t))+\kappa\right] V^{\frac{1}{2}}(u(t))
\end{aligned}
$$

$$
\begin{aligned}
\mathbb{E}\left[\| f_{v}(\hat{u}(t))\right. & \left.-\hat{f}_{v}(\hat{u}(t)) \|_{N}^{2} \mid u(t)\right] \\
& \leq K_{10}(1+V(u(t))) h_{k}^{2} \\
& \leq K_{10} \tilde{V}(u(t)) h_{k}^{2} .
\end{aligned}
$$

where

$$
\begin{aligned}
K_{10} & =8 \cdot\left(\kappa^{2}+2 \sigma^{4} N^{4} \pi^{4}\left(\frac{1}{l_{x}^{2}}+\frac{1}{l_{y}^{2}}\right)^{4}+2 a_{2}^{2} V(u(t))\right) \\
& {\left[\left(2 \sigma^{4} N^{4} \pi^{4}\left(\frac{1}{l_{x}^{2}}+\frac{1}{l_{y}^{2}}\right)^{4}+2 a_{2} V(u(t))\right)\left(\frac{2}{a_{2}}\right)^{\frac{1}{4}} V^{\frac{3}{2}}(u(t))+\kappa\right], }
\end{aligned}
$$

therefore

$$
\begin{aligned}
\int_{t}^{t+h} \mathbb{E}\left[\| f_{v}(\hat{u}(t))\right. & \left.-\hat{f}_{v}(\hat{u}(t)) \|_{N}^{2} \mid u(t)\right] d s \\
& \leq K_{10} \tilde{V}(u(t)) h_{k}^{3} \\
& \leq K_{10} \tilde{V}(u(t)) h_{k}^{2} .
\end{aligned}
$$

To complete the proof, we have to simplify the second part of the right side of inequality (24) which is

$$
\begin{aligned}
\| \int_{t}^{t+h}\left[g_{v}(u(s))\right. & \left.-\hat{g}_{v}(u(t))\right] d W(s) \|_{N}^{2} \\
& \leq C_{B} \int_{t}^{t+h}\left\|g_{v}(u(s))-\hat{g}_{v}(u(t))\right\|_{N}^{2} d s
\end{aligned}
$$

but from [10] we know that

$$
\begin{aligned}
& \left\|\int_{t}^{t+h}\left[g_{v}(u(s))-\hat{g}_{v}(u(t))\right] d W(s)\right\|_{N}^{2} \\
& \leq C_{B} \int_{t}^{t+h}\left\|g_{v}(u(s))-g_{v}(u(t))\right\|_{N}^{2} d s \\
& +C_{B} \int_{t}^{t+h}\left\|g_{v}(u(t))-\hat{g}_{v}(u(t))\right\|_{N}^{2} d s .
\end{aligned}
$$


We start with the first part of the right side of inequality (35) which hence

is

$\int_{t}^{t+h}\left\|g_{v}(u(s))-g_{v}(u(t))\right\|_{N}^{2} d s$

$\leq \int_{t}^{t+h}\left\|b_{1}\left(\|u(s)\|_{l^{2}}-\|u(t)\|_{l^{2}}\right)+b_{2}\left(\|v(s)\|_{l^{2}}-\|v(t)\|_{l^{2}}\right)\right\|_{N}^{2} d s$

$\leq \int_{t}^{t+h}\left[2 b_{1}^{2}\left(\|u(s)\|_{l^{2}}-\|u(t)\|_{l^{2}}\right)^{2}+2 b_{2}^{2}\left(\|v(s)\|_{l^{2}}-\|v(t)\|_{l^{2}}\right)^{2}\right] d s$ $\leq \int_{t}^{t+h} \max \left\{2 b_{1}^{2}, 2 b_{2}^{2}\right\}\left[\left(\|u(s)\|_{l^{2}}-\|u(t)\|_{l^{2}}\right)^{2}+\left(\|v(s)\|_{l^{2}}-\|v(t)\|_{l^{2}}\right)^{2}\right] d s$

$\leq \int_{t}^{t+h} 2 \max \left\{2 b_{1}^{2}, 2 b_{2}^{2}\right\}\left[\|u(\gamma)\|_{l^{2}}^{2}|s-t|+\|v(\gamma)\|_{l^{2}}^{2}|s-t|\right] d s$ $\leq \max \left\{2 b_{1}^{2}, 2 b_{2}^{2}\right\} V(u(t)) h_{k}^{2}$

thus

$$
\begin{aligned}
\int_{t}^{t+h} \mathbb{E} \| g_{v}(u(s)) & -g_{v}(u(t)) \|_{N}^{2} d s \\
& \leq K_{11} V(u(t)) h_{k}^{2} \leq K_{11} \tilde{V}(u(t)) h_{k}^{2}
\end{aligned}
$$

where $K_{11}=\max \left\{2 b_{1}^{2}, 2 b_{2}^{2}\right\}$. Also, the second part of the right side of inequality (35), we start with

$$
\begin{aligned}
\mathbb{E} & {\left[\left\|g_{v}(u(t))-\hat{g}_{v}(u(t))\right\|_{N}^{2}\right] } \\
= & h_{k} \mathbb{E}\left[\left\|\frac{\left[\kappa+h_{k} F(u(t))\right]\left[b_{0}+b_{1}\|u(t)\|_{l^{2}}+b_{2}\|v(t)\|_{l^{2}}\right]}{1+h_{k} \kappa+h_{k}^{2} F(u(t))}\right\|_{N}^{2}\right] \\
\leq & h_{k}\left(\mathbb{E}\left\|\kappa+h_{k} F(u(t))\right\|_{N}^{4}\right)^{\frac{1}{2}}\left(\mathbb{E}\left\|b_{0}+b_{1}\right\| u(t)\left\|_{l^{2}}+b_{2}\right\| v(t)\left\|_{l^{2}}\right\|_{N}^{4}\right)^{\frac{1}{2}} \\
\leq & 81 h_{k}\left(\mathbb{E}\left[\kappa^{4}+h_{k}^{4} \sigma^{4} N^{4} \pi^{4}\left(\frac{1}{l_{x}^{2}}+\frac{1}{l_{y}^{2}}\right)^{4}+a_{2}^{4}\|u(t)\|_{l^{2}}^{8}\right]\right)^{\frac{1}{2}} \\
& \cdot\left(\mathbb{E}\left[b_{0}^{4}+b_{1}^{4}\|u(t)\|_{l^{2}}^{4}+b_{2}^{4}\|v(t)\|_{l^{2}}^{4}\right]\right)^{\frac{1}{2}},
\end{aligned}
$$

$$
\begin{aligned}
& \mathbb{E}\left[\left\|g_{v}(u(t))-\hat{g}_{v}(u(t))\right\|_{N}^{2} \mid u(t)\right] \\
& \leq 81 h_{k}\left(\kappa^{4}+h_{k}^{4} \sigma^{4} N^{4} \pi^{4}\left(\frac{1}{l_{x}^{2}}+\frac{1}{l_{y}^{2}}\right)^{4}+a_{2}^{4} \mathbb{E}\|u(t)\|_{l^{2}}^{8}\right)^{\frac{1}{2}} \\
& \cdot\left(b_{0}^{4}+b_{1}^{4} \mathbb{E}\|u(t)\|_{l^{2}}^{4}+b_{2}^{4} \mathbb{E}\|v(t)\|_{l^{2}}^{4}\right)^{\frac{1}{2}} \\
& \leq 81 h_{k}\left(\kappa^{4}+h_{k}^{4} \sigma^{4} N^{4} \pi^{4}\left(\frac{1}{l_{x}^{2}}+\frac{1}{l_{y}^{2}}\right)^{4}+4 a_{2}^{2} \mathbb{E} V^{2}(u(t)) \mid u(t)\right)^{\frac{1}{2}} \\
& \cdot\left(b_{0}^{4}+\frac{2 b_{1}^{4}}{a_{2}} \mathbb{E} V(u(t))\left|u(t)+b_{2}^{4} \mathbb{E} V^{2}(u(t))\right| u(t)\right)^{\frac{1}{2}} \\
& \leq 81 h_{k}\left(\kappa^{4}+h_{k}^{4} \sigma^{4} N^{4} \pi^{4}\left(\frac{1}{l_{x}^{2}}+\frac{1}{l_{y}^{2}}\right)^{4}+4 a_{2}^{2} V^{2}(u(t))\right)^{\frac{1}{2}} \\
& \cdot\left(b_{0}^{4}+\frac{2 b_{1}^{4}}{a_{2}} V(u(t))+b_{2}^{4} V^{2}(u(t))\right)^{\frac{1}{2}} \\
& \leq 81 h_{k}\left(\kappa^{2}+h_{k}^{2} \sigma^{2} N^{2} \pi^{2}\left(\frac{1}{l_{x}^{2}}+\frac{1}{l_{y}^{2}}\right)^{2}+2 a_{2} V(u(t))\right) \\
& \cdot\left(b_{0}^{2}+\frac{\sqrt{2} b_{1}^{2}}{\sqrt{a_{2}}}(V(u(t)))^{\frac{1}{2}}+b_{2}^{2} V(u(t))\right) \\
& \leq 81\left(\kappa^{2}+h_{k}^{2} \sigma^{2} N^{2} \pi^{2}\left(\frac{1}{l_{x}^{2}}+\frac{1}{l_{y}^{2}}\right)^{2}+2 a_{2}(V(u(t)))^{\frac{1}{2}}\right) \\
& \cdot\left(b_{0}^{2}+\frac{\sqrt{2} b_{1}^{2}}{\sqrt{a_{2}}}+b_{2}^{2}(V(u(t)))^{\frac{1}{2}}\right)(V(u(t)))^{\frac{1}{2}} h_{k} \\
& \leq 81\left(\kappa^{2}+h_{k}^{2} \sigma^{2} N^{2} \pi^{2}\left(\frac{1}{l_{x}^{2}}+\frac{1}{l_{y}^{2}}\right)^{2}+2 a_{2}(V(u(t)))^{\frac{1}{2}}\right) \\
& \cdot\left(b_{0}^{2}+\frac{\sqrt{2} b_{1}^{2}}{\sqrt{a_{2}}}+b_{2}^{2}(V(u(t)))^{\frac{1}{2}}\right)(1+V(u(t))) h_{k}
\end{aligned}
$$

thus

$$
\mathbb{E}\left[\left\|g_{v}(u(t))-\hat{g}_{v}(u(t))\right\|_{N}^{2} \mid u(t)\right] \leq K_{12} \tilde{V}(u(t)) h_{k}
$$

where

$$
\begin{aligned}
K_{12}= & 81\left(\kappa^{2}+h_{k}^{2} \sigma^{2} N^{2} \pi^{2}\left(\frac{1}{l_{x}^{2}}+\frac{1}{l_{y}^{2}}\right)^{2}+2 a_{2}(V(u(t)))^{\frac{1}{2}}\right) \\
& \cdot\left(b_{0}^{2}+\frac{\sqrt{2} b_{1}^{2}}{\sqrt{a_{2}}}+b_{2}^{2}(V(u(t)))^{\frac{1}{2}}\right)
\end{aligned}
$$

therefore

$\int_{t}^{t+h} \mathbb{E}\left[\left\|g_{v}(u(t))-\hat{g}_{v}(u(t))\right\|_{N}^{2} \mid u(t)\right] d s \leq K_{12} \tilde{V}(u(t)) h_{k}^{2}$.

Thus by using inequalities (31), (34), (36), and (38) we can write inequality (15) as

$$
\begin{aligned}
\mathbb{E}\left[\| v_{n, m}(t+h)\right. & \left.-\hat{v}_{n, m}(t+h) \|_{N}^{2} \mid \hat{u}(t)\right] \\
& \leq\left[4\left(K_{9}+K_{10}\right)+2 C_{B}\left(K_{11}+K_{12}\right)\right] \tilde{V}(u(t)) h_{k}^{2} \\
& \leq K_{v_{2}}^{c} \tilde{V}(u(t)) h_{k}^{2}
\end{aligned}
$$

where $K_{v_{2}}^{c}=4\left(K_{9}+K_{10}\right)+2 C_{B}\left(K_{11}+K_{12}\right)$. Thus

$$
\begin{aligned}
\left(\mathbb{E}\left[\left\|v_{n, m}(t+h)-\hat{v}_{n, m}(t+h)\right\|_{N}^{2} \mid \hat{u}(t)\right]\right)^{\frac{1}{2}} & \leq K_{v_{2}}^{c}(\tilde{V}(u(t)))^{\frac{1}{2}} h_{k} \\
& \leq K_{v_{2}}^{c} \tilde{V}(u(t)) h_{k} .(40)
\end{aligned}
$$

III. CONCLUSION

In this article we prove that the linear-implicit Euler method is mean square consistent with rate $r_{0}=2$ on nonlinear stochastic wave equations in 2 dimensions. In the future, we will continue to show that the LIEM is mean square and asymptotic stability, and convergence. 
Also, we will study the stability and mean square consistency of the solution of stochastic heat equation, Burger equation.

\section{REFERENCES}

[1] L. Arnold, Stochastic Differential Equations: Theory and Applicants, John Wiley and Sons, Inc., New York, 1974.

[2] P.L. Chow, Stochastic Partial Differential Equations. Chapman and Hall/CRC, Boca Raton, 2007.

[3] R. Dalang and N.E. Frangos, The stochastic wave equation in two spatial dimensions. Ann. Probab. 26 (1998), 187-212.

[4] G. Da Prato and J. Zabzcyk, Stochastic Equations in Infinite dimensions, Cambridge University Press, Cambridge, 1992.

[5] A. Friedman, Stochastic Differential Equations and Applications, Dover Publications, Inc. Mineola, New York, 1975.

[6] T. C. Gard, Introduction to Stochastic Differential Equations, Marcel Dekker, Basel, 1988.

[7] W. Grecksch and C. Tudor, Stochastic Evolutions: A Hilbert Space Approach, Akademie-Verlag, Berlin, 1995.

[8] Schurz, H., and Hazaimeh, H. M. (2014). Existence, uniqueness, and stability of stochastic wave equation with cubic nonlinearities in two dimensions. Journal of Mathematical Analysis and Applications, 418(2), 775-795.

[9] Hazaimeh, H. M. (2012). Stochastic Wave Equations With Cubic Nonlinearities in Two Dimensions.

[10] H. M. Hazaimeh, Local Mean Consistency on Numerical solution of stochastic wave equation with cubic nonlinearities in two dimensions. Applied Institute of Physics, 2017.

[11] Desmond Higham, Mean?square and asymptotic stability of the stochastic theta method, SIAM J. Numer. Anal. , 38 (2000), 753 ?769

[12] Higham, D.J., Mao, X., Stuart, A.M.: Strong convergence of Euler-like methods for nonlinear stochastic differential equations. SIAM J. Numer. Anal. 40, 1041?1063 (2002)

[13] I. Karatzas and S.E. Schreve, Brownian Motion and Stochastic Calculus, Second Edition. Springer-Verlag, New York, 1991.

[14] B. Oksendal, Stochastic Differential Equations: An Introduction With applications, Six Edition, Springer-Verlag, Berlin, 2003.

[15] A. Millet and M. Sanz-Solé, A stochastic wave equation in two space dimension: Smootheness of the law, Ann. Probab. 27 1999, 803-844.

[16] X. Mao, Stochastic Differential Equations and Applications, Horwood Publishing, Chichester, 1997.

[17] H. Schurz, Nonlinear Stochastic heat equations with cubic nonlinearity and Additive Q-regular Noise in $\mathbb{R}^{1}$, Electronic Journal of Differential Equations, Conf. 19 (2010), 221-233.

[18] H. Schurz, Stochastic wave equations with cubic nonlinearity and Qregular Additive Noise in $\mathbb{R}^{2}$, Discrete and Continuous Dynamical Systems, Suppl. Vol. 2011 (2011), 1299-1308.

[19] A. N. Shiryaev, Probability (2nd edition), Springer-Verlag, Berlin, 1996.

[20] J. B. Walsh, An introduction to stochastic partial differential equations, École d'été de probabilités de Saint-Flour, XIV-1984, 265-439, Lecture Notes in Math. 1180, Springer-Verlag, Berlin, 1986. 